\title{
Harmonious Coloring for Subdivision of Certain Classes of Graph
}

\author{
Franklin Thamil Selvi. M.S, A.Amutha
}

\begin{abstract}
For any graph $G, \chi_{-} h(G)$ is the extremely small number of colors used in a graph such that no two nearby vertices gets a similar color and every mix of color appears to be together on atmost one edge. In this article we have decided the harmonious chromatic number of subdivision of certain groups of graphs.
\end{abstract}

\section{INTRODUCTION}

Graph coloring problem is of enthusiasm for its applications. Coloring problems manages the crucial issues of parceling an arrangement of articles into classes as indicated by specific tenets. Time tabling, sequencing and booking issues in their numerous structures, are essentially of this nature. A wonderful method for coloring a graph legitimately is harmonious coloring [1-7]. This issue has potential effectiveness in correlation systems, for example transportation systems, PC systems, piloting route organize structure, satellite route structure, radio route structure and so on. Harmonious coloring was first presented by Frank Harray and Plantholt in 1982 [6]. Anyway the best possible meaning of this thought is because of Lee Hopcroft and Krishnamoorty [7]. The minimum number of harmonious coloring of a few distinct groups of graphs has been found by various creators. The boundary of this problem might be considered as an edge accepting an interesting shading pair [3,5]. It was appeared by Hopcroft and Krishnamoorthy that the issue of deciding this problem is NP-hard $[1,4]$. Despite the fact that there are various outcomes and exchanges on our concern, the greater part of them bargain just with estimation results [2,3]. So creators began taking a shot at central, line and total of some recognized graphs[2].One idea that infests all of graph theory is that of separation and separation is utilized in isomorphism testing, diagram task, outside issue on network and breath. Hence subdivision of any graph is acquired by subdividing each edge of $G$ precisely once. Motivated by these issues we

have determined the subdivision of certain groups of graphs like complete bipartite and shell graph. Also we have characterised the results with matching and edge covering number.

\section{MAIN RESULTS}

\subsection{Subdivision of complete bipartite graphs}

In this segment, we decide the harmonious chromatic number of subdivision of Complete Bipartite Graph .

Revised Manuscript Received on October 25, 2019.

Franklin Thamil Selvi.M.S, Department of Mathematics, Sathyabama Institute of Science and Technology, India. thamizanand@gmail.com

A.Amutha, Department of Mathematics, The American College, Madurai, India. amuthajo@gmail.com

\section{Perception 2.1.1}

Let $G$ be $K_{m, n}$ at that point for $\mathrm{m}=\mathrm{n}, G$ possess a perfect matching and $\alpha^{*}(\mathrm{G})=\mathrm{n}$.

\section{Perception 2.1.2}

Let $G$ be $K_{m_{n} n}$ at that point for $\mathrm{m}=\mathrm{n} \quad \chi_{n}\left(K_{m_{n} n}\right)=2 \alpha^{\circ}$.

\section{Perception 2.1.3}

Let $G$ be $K_{m_{n} n}$ at that point for $\mathrm{m}=\mathrm{n} \chi_{h}\left(K_{m_{j} n}\right)=2 \alpha^{\circ}$ if and just if $G$ has no unsaturated nodes.

\section{Perception 2.1.4}

Let $G$ be $K_{m, n}$ at that point for $\mathrm{m} \neq \mathrm{n}$ and $\mathrm{n}>\mathrm{m}, G$ does not posses perfect matching and $\alpha^{x}(G)=\min \left(m_{x} n\right)$.

\section{Perception 2.1.5}

Let $G$ be $K_{m_{n} n}$ at that point for $\mathrm{m} \neq \mathrm{n}$ and $\mathrm{n}>\mathrm{m}, G$ does not possess perfect matching and there persist $\mathrm{m}-\mathrm{n}$ unsaturated nodes.

\section{Theorem 2.1.6}

Let $G$ be $K_{m n}$ at that point for $\mathrm{m} \neq \mathrm{n}$ and $\mathrm{n}>\mathrm{m}$, $\gamma_{n}\left(K_{m_{n} n}\right)=\alpha(G)+\max \left(m_{s} n\right)$.

\section{Proof}

Let $G$ be $K_{m n}$ with $\mathrm{n}+\mathrm{m}$ nodes and mn lines. We allot color to these nodes as pursues. The maximum degree of $K_{m n}$ is n, then we require $\mathrm{n}+1$ colors to color these nodes, and rest of $\mathrm{m}-1$ nodes are colored with $\mathrm{m}-1$ colors. Obviously we need $\mathrm{n}+1+\mathrm{m}-1$ colors. That is $\mathrm{m}+\mathrm{n}$ colors where $\mathrm{m}$ is $\mathrm{m}$ is $(\mathrm{m}, \mathrm{n})$ and $\mathrm{n}$ is $\max (\mathrm{m}, \mathrm{n})$. Hence $\chi_{\mathrm{h}}\left(K_{\mathrm{m}_{n} \mathrm{n}}\right)=\alpha(\mathrm{G})+\max \left(\mathrm{m}_{\mathrm{n}} \mathrm{n}\right)$.

We demonstrate the outcome by induction.

For $m=1$ and $n>m$ the outcome is self evident. Accept that the hypothesis holds for $\mathrm{m}=\mathrm{m}$ and $\mathrm{n}>\mathrm{m}$, then $\chi_{\mathrm{h}}\left(K_{m_{n} n}\right)=\alpha(\mathrm{G})+\max \left(\mathrm{m}_{\mathrm{n}} \mathrm{n}\right)$.

Let us substantiate for $m=m+1$ and $n>m$.

Observe $\left(K_{m+1, n}\right)$. A Complete Bipartite graph $\left(K_{m+1 n}\right)$ is obtained by including one node and $\mathrm{n}$ lines to $\left(K_{m n}\right)$. Expelling such node in $\left(K_{m+1 n}\right)$, we get a subgraph $G$ ' which is nothing but $\left(K_{m_{n} n}\right)$. Thus by presumption we have 


\section{Harmonious Coloring for Subdivision of Certain Classes of Graph}

$\chi_{h}\left(K_{m, n}\right)=\alpha^{\prime}(G)+\max \left(m_{n} n\right)$. Now by including the evacuation nodes, the maximum degree does not change. The maximum matching number will be increased by one that is $\min (m+1, n)$ and $\max (m+1, n)$ will be same as $\max (m, n)$.

Therefore $\gamma_{h}\left(K_{m+1 n}\right)=\alpha\left(K_{m_{n} n}\right)+1+\max \left(m_{s} n\right)$

$$
=\alpha\left(K_{m+1, n}\right)+\max \left(m+1_{s} n\right)
$$

Hence the proof.

\section{Theorem 2.1.7}

Let $G$ be $K_{m, n}$ at that point for $\mathrm{m} \neq \mathrm{n}$ and $\mathrm{n}>\mathrm{m}$, $\chi_{h}\left(K_{m_{n} n}\right)=\alpha(G)+\max \left(m_{s} n\right)$ if and just if $G$ possess $n-$ $\mathrm{m}$ unsaturated nodes.

\section{Proof}

Assume that $\chi_{h}\left(K_{m_{n} n}\right)=\alpha(G)+\max \left(m_{s} n\right)$, by the above outcome $\alpha^{\prime}(\mathrm{G})=\min \left(\mathrm{m}_{\mathrm{s}} \mathrm{n}\right)$. At that point $\alpha^{\prime}(G)=m$ covers $2 \mathrm{~m}$ nodes, no where equivalent to the number of nodes in G. Subsequently $G$ does not possess a perfect matching. At that point there persist unsaturated nodes say $n-m$ by Perception 2.1.5.

On the other hand, assume $\mathrm{G}$ has $n-m$ unsaturated nodes then $G$ does not possess perfect matching by the above outcome $\alpha^{\prime \prime}(G)=\min \left(m_{s} n\right)$. By theorem 2.1.6 we realize that $\chi_{n}\left(K_{m, n}\right)=\alpha(G)+\max (m, n)$.

Hence the proof.

\section{Perception 2.1.8}

Let $G$ be $S\left(K_{m, n}\right)$ at that point for $\mathrm{m}=\mathrm{n}=2, G$ possess a perfect matching.

\section{Perception 2.1.9}

Let $G$ be $S\left(K_{m, n}\right)$ at that point for $m=n=2, \alpha^{\circ}(G)=2 n$.

\section{Perception 2.1.10}

Let $G$ be $S\left(K_{m, n}\right)$ at that point for $\mathrm{m}$ or $\mathrm{n}$ is equal to one $G$ does not possess perfect matching and $\alpha^{\prime}(G)=\max (m, n)$.

\section{Perception 2.1.11}

Let $G$ be $S\left(K_{m n}\right)$ at that point for $\mathrm{m}$ or $\mathrm{n}$ is equal to one $G$ does not possess perfect matching and there is only one unsaturated node.

\section{Perception 2.1.12}

Let $G$ be $S\left(K_{m, n}\right)$ at that point for $m>2$ and $n>m, G$ does not possess perfect matching and $\alpha(G)=m+n$.

\section{Perception 2.1.13}

Let $G$ be $S\left(K_{m, n}\right)$ at that point for $\mathrm{m} \geq 2$ and $\mathrm{n} \geq 2, G$ does not possess perfect matching and there are $n(m-1)-m$ unsaturated nodes.

\section{Theorem 2.1.14}

$$
\chi_{h}\left\{S\left(K_{m_{n} n}\right)\right\}=\Delta\left\{S\left(K_{m_{n} n}\right)\right\}+1 \text { for } m>0 \text { and } n>2 m
$$

\section{Proof}

Let $\mathrm{G}$ be $K_{\mathrm{m}_{n} \mathrm{n}}$ with $\mathrm{n}+\mathrm{m}$ nodes and $\mathrm{mn}$ lines. Now by the definition of subdivision, subdividing each line precisely once, the number of nodes in subdivision of complete bipartite graph is $n+m+m n$. We allocate color to these nodes as pursues. The maximum degree of $S\left(K_{m, n}\right)$ is $n$, hence we need $n+1$ colors to color these nodes, and the remaining $m(n$ +1 ) nodes are colored with the current colors. Subsequently no new colors are required. Henceforth we need $n+1$ colors Hence $\chi_{h}\left(S\left(K_{m_{n} n}\right)\right)=\Delta\left\{S\left(K_{m_{n} n}\right)\right\}+1$.

We can informally illustrate by induction on $\mathrm{n}$ and $\mathrm{m}$.

For $m=1$ and $n>2 m$ the result is evident. Presume that the theorem holds for $\mathrm{m}=\mathrm{m}$ and $\mathrm{n}>2 \mathrm{~m}$, then $\gamma_{h}\left\{S\left(K_{m_{n}}\right)\right\}=\Delta\left\{S\left(K_{m_{n} n}\right)\right\}+1$

Let us substantiate by induction for $m=m+1$ and $n>m$.

Observe $S\left(K_{m+1 n}\right)$. A Complete Bipartite graph $\left(K_{m+1 n}\right)$ is obtained by including one node and $\mathrm{n}$ lines to $\left(K_{m, n}\right)$. By the definition of subdivision the $\mathrm{n}$ edges are subdivided by another node. Consequently $S\left(K_{m+1 n}\right)$ is obtained by attaching $\mathrm{n}+1$ vertices to $S\left(K_{m_{n} n}\right)$. Expelling such vertex in $S\left(K_{m+1, n}\right)$, we obtain a subgraph $\mathrm{G}^{\prime}$ which is nothing but $S\left(K_{m_{n} n}\right)$. Subsequently by presumption we have $\gamma_{h}\left\{S\left(K_{m_{n} n}\right)\right\}=\Delta\left\{S\left(K_{m_{n} n}\right)\right\}+1$ Now by including the expulsion nodes, the maximum degree does not change.

Therefore $\gamma_{h}\left\{S\left(K_{m+1, n}\right)\right\}=\Delta\left\{S\left(K_{m+1, n}\right)\right\}+1$

Hence the proof.

\section{Theorem 2.1.15}

Let $\mathrm{G}$ be a $S\left(K_{m n}\right)$, at that point for $\mathrm{n}>2 \mathrm{~m}$ and $\mathrm{n}>\mathrm{m}$ $\chi_{n}\left\{S\left(K_{m_{n}}\right)\right\}=\alpha\left\{S\left(K_{m_{n} n}\right)+1\right.$ if and just if $\mathrm{G}$ possess $n(m-1)-m$ unsaturated vertices, where $\alpha^{\prime}$ is the maximum matching number.

\section{Proof}

Assume that $\gamma_{h}\left\{S\left(K_{m_{n} n}\right)\right\}=\alpha\left\{S\left(K_{m_{n} n}\right)+1\right.$, by the above result $\alpha^{\prime}(\mathrm{G})=\mathrm{n}$ and $\Delta\left\{S\left(K_{\mathrm{m}_{n}}\right)\right\}=n$. Then $\alpha^{\prime}(\mathrm{G})=\mathrm{n}$ covers $2 \mathrm{n}$ nodes, no where equivalent to the number of vertices in $G$. Hence $G$ does not possess a perfect matching. At that point there persist unsaturated nodes say $n(m-1)-m$ by Perception 2.1.13.

On the other hand, assume $G$ has $n(m-1)-m$ unsaturated nodes then $\mathrm{G}$ does not possess perfect matching by the above result $\alpha^{\prime}(G)=$ n. By theorem 2.1.14 we know that

$$
\begin{aligned}
& \chi_{h}\left\{S\left(K_{m_{n} n}\right)\right\}=\Delta\left\{S\left(K_{m_{n} n}\right)\right\}+1 \text { for } n \geq 2 m \text { and } n>m . \\
& \text { Hence } \chi_{h}\left\{S\left(K_{m_{n} n}\right)\right\}=\alpha\left\{S\left(K_{m_{n} n}\right)+1 .\right.
\end{aligned}
$$

Hence the proof. 


\subsection{Subdivision of shell graphs}

In this segment, we decide the harmonious coloring of $S\{C(n, n-3)\}$.

\section{Definition 2.2.1}

A shell graph of size $\mathrm{n} \geq 4$ indicated by $C(n, n-3)$ is a graph got from cycle $C_{n}\left(v_{0}, v_{1}, v_{2}, \ldots \ldots, v_{n-1}\right)$ by attaching $\mathrm{n}-3$ consecutive chords episode with a familiar vertex $v_{0}$ (say) called apex of the shell graph.

\section{Perception 2.2.2}

Let $\mathrm{G}$ be a $S\{C(n, n-3)\}$ at that point, $G$ does not possess a perfect matching and $\alpha^{t}(\mathrm{G})=\mathrm{n}+1$.

\section{Perception 2.2.3}

Let $\mathrm{G}$ be a $S\left\{C\left(n_{n} n-3\right)\right\}$ at that point, $\mathrm{G}$ does not possess a perfect matching and it has $\mathrm{n}-3$ unsaturated node.

\section{Theorem 2.2.4}

$$
\begin{aligned}
& \chi_{h}\{S[C(n, n-3)]\}=\Delta\{S[C(n, n-3)]\}+\Delta\{C(n, n- \\
& 3)\}-1
\end{aligned}
$$

\section{Theorem 2.2.5}

Let $\mathrm{G}$ be a $S\{C(n, n-3)\}$, at that point

$$
\chi_{h}\{S[C(n, n-3)]\}=\alpha^{s}\{S[C(n, n-3)]\}+\Delta\{C(n, n-
$$

3)\}

if and just if $G$ has $n-3$ unsaturated nodes, where $\alpha^{\prime}$ is the maximum matching number.

\section{CONCLUSION}

Assume

$$
\chi_{h}\{S[C(n, n-3)]\}=\alpha^{n}\{S[C(n, n-3)]\}+\Delta\{C(n, n-
$$

3)\}

by the above outcome $\alpha^{\prime}(\mathrm{G})=\mathrm{n}+1$ and $\Delta\{C(n, n-3)\}=n$. Then $\alpha^{\prime}(G)=\mathrm{n}+1$ covers $2(\mathrm{n}+1)$ nodes, no where equivalent to the number of nodes in $\mathrm{G}$. Hence $\mathrm{G}$ does not possess a perfect matching. At that point there persist unsaturated nodes say $n-3$ by Perception 2.2.3.

On the other hand, suppose $\mathrm{G}$ has $\mathrm{n}-3$ unsaturated nodes then $\mathrm{G}$ does not have perfect matching by the above outcome $\alpha^{n}(\mathrm{G})=\mathrm{n}+1$. By theorem 2.2.4 we know that $\chi_{h}\{S[C(n, n-3)]\}=\Delta\{S[C(n, n-3)]\}+\Delta\{C(n, n-$ 3) $3-1$

$X_{h}\{S[C(n, n-3)]\}=\alpha^{x}\{S[C(n, n-3)]\}+\Delta\{C(n, n-$ 3) 3

\section{REFERENCES}

1. K. Asdre, K. Ioannidou and S. D Nikolopoulos, "The harmonious coloring problem is NP-complete for interval and permutation graphs," Discrete Applied Math, vol. 155, pp. 2377-2382, (2007).
2. Bharati Rajan, Indra Rajan, and D. Franci Xavier, "Harmonious coloring of Honeycomb networks," Journal of Computer and Mathematical Sciences, vol. 2, pp. 882-887, (2011).

3. D. Campbell and K. J. Edwards, "A new lower bound for the harmonious chromatic number," Australasian Journal of Combinatorics, vol. 29, pp. 99-102, (2004).

4. K. J. Edwards and C. J. H. Diarmid, "The complexity of harmonious coloring for trees," Discrete Applied Math, vol. 57, pp. 133-144, (1995).

5. K. J. Edwards and C. J. H. Diarmid, "New upper bounds on harmonious coloring," Journal Of Graph Theory, vol. 18, pp. 257-267, (1994).

6. O. Frank, F. Harary and M. Plantholt, "The line distinguishing chromatic number of a graph," Ars Combinatorics, vol. 14, pp. 241-252, (1982).

7. J. E. Hopcroft and Krishnamoorthy, "On the harmonious coloring of graph," J. Algebraic and discrete Methods, vol. 4, pp. 306 - 31, (1983). 